\title{
Pilotierung einer Ultrafiltrationsanlage an einem Uferfiltratstandort in Österreich - Untersuchungen der bakteriellen Zellzahlen und der biologischen Stabilität im Filtrat
}

\author{
P. Proksch - D. J. Kerschbaumer · C. Schönher - M. Zunabovic-Pichler · E. Mayr · R. Perfler \\ Online publiziert: 7. Oktober 2020 \\ (c) Der/die Autor(en) 2020
}

Zusammenfassung Ultrafiltration stellt eine immer öfter eingesetzte Aufbereitungsmethode für Trinkwasser dar, die insbesondere durch den zuverlässigen Rückhalt von Mikroorganismen ohne Veränderung der Zusammensetzung des Wassers hervorsticht. Aufgrund der komplexen Betriebsweise empfiehlt es sich jedoch, solche Anlagen vor der großtechnischen Herstellung durch eine Pilotierung $\mathrm{zu}$ testen. In diesem Artikel werden die Ergebnisse eines solchen Pilotierungsversuchs vorgestellt, wobei ein besonderes Augenmerk auf die durch Durchflusszytometrie bestimmten Zellzahlen und die mittels Wachstumsversuchen ermittelte Biostabilität gelegt wird. Dabei zeigte sich, dass im Filtrat einerseits die Zellzahlen nicht vollständig gegen Null gingen, was vermutlich auf filtratseitige Verunreinigungen zurückzuführen ist, und andererseits, dass diese wenigen Zellen durch das Vorhandensein zusätzlicher Nährstoffe ein starkes Wachstum aufwiesen und dadurch die Biostabilität maßgeblich beeinflusst wurde. Da in der weiteren Speicherung und Verteilung von Trinkwasser mikrobielles Wachstum so gut wie möglich zu vermeiden ist, ist es wichtig, unter solchen wachstumsfördernden Bedingungen

DI P. Proksch $(\bowtie)$.

DI D. J. Kerschbaumer, B.Sc. .

DI C. Schönher .

DI Dr. M. Zunabovic-Pichler .

PD DI Dr. R. Perfler

Institut für Siedlungswasserbau,

Industriewasserwirtschaft

und Gewässerschutz (SIG),

Universität für Bodenkultur Wien, Muthgasse 18, 1190 Wien, Österreich philipp.proksch@boku.ac.at

DI E. Mayr

MA 31 - Wiener Wasser, Grabnergasse 4-6, 1061 Wien, Österreich entsprechende Maßnahmen zu setzen, die ein biologisch stabiles System gewährleisten.

Schlüsselwörter Ultrafiltration . Uferfiltration - Durchflusszytometrie . Biologische Stabilität

Piloting an ultrafiltration plant at a river bank filtration site in Austria-investigating bacterial cell counts and the biologic stability in the filtrate

Abstract Ultrafiltration is a treatment method for drinking water that is being used more and more frequently. It stands out, for its reliable retention of microorganisms without changing the composition of the water. Due to the complex mode of operation, however, it is advisable to test such plants by means of a pilot test prior to large-scale construction. In this article the results of such a pilot test are presented, with special emphasis on the cell counts determined by flow cytometry and the biostability determined by growth experiments. It was shown, that in the filtrate on the one hand the cell numbers did not completely approach zero, which is probably due to filtrate side impurities. On the other hand, that these few cells showed strong growth due to the presence of additional nutrients, which significantly influenced the biostability. Since microbial growth should be avoided as much as possible in the further storage and distribution of drinking water, it is important to further take appropriate measures to ensure a biologically stable system under such growth-promoting conditions.

Keywords Ultrafiltration - River bank filtration · Flow cytometry $\cdot$ Biologic stability

\section{Einleitung}

1.1 Ultrafiltration

Ultrafiltration findet auch in Österreich gerade in den letzten Jahren immer öfter Anwendung in der Aufbereitung von Trinkwasser. Bei aufwendigeren Aufbereitungsanlagen findet sie sich oftmals inmitten der Aufbereitungskette zum Beispiel als Vorstufe zur Umkehrosmose. Aber vermehrt kommt die Ultrafiltration auch als einzige Aufbereitungsstufe vor der Desinfektion zum Einsatz - insbesondere dann, wenn nur eine Abtrennung partikulärer Stoffe, beispielsweise vor einer UV-Desinfektion, gewünscht ist.

Gemeinsam mit der Mikrofiltration, der Nanofiltration und der Umkehrosmose wird die Ultrafiltration $\mathrm{zu}$ den Membrantechnologien gezählt. Die genannten Technologien werden zwar meist anhand ihrer Porengröße unterschieden, aber je nach Autor liegen diese Angaben durchaus auseinander. Laut DVGW ist bei der Ultrafiltration ein Rückhalt von 99,99\% (4 log-Stufen) von Partikeln zwischen 20 und $30 \mathrm{~nm}$ möglich, während die Mikrofiltration Partikel kleiner $100 \mathrm{~nm}$ unzureichend zurückgehalten werden (DVGW W 213-5 2019). Während bei der Nanofiltration und der Umkehrosmose aufgrund der noch kleineren Porengröße mit weitaus höheren Drücken (bis zu 100 bar bei vollständiger Entsalzung) gearbeitet werden muss, kann bei der Ultrafiltration in der Regel mit Drücken unter einem bar gearbeitet werden (Crittenden et al. 2012).

Die Größe der Poren wirkt sich auch direkt auf die zurückgehaltenen Stoffe aus, da ausschließlich eine physikalische Abtrennung anhand der Größe passiert und keine zusätzlichen Prozesse, wie zum Beispiel Adsorption und Absorption, stattfinden. Bei der Ultrafil- 
tration ist aufgrund der Porengröße in der Regel ein vollständiger Rückhalt von Bakterien, deren kleinste Vertreter zwischen 100 und $200 \mathrm{~nm}$ groß sind, und ein (zumindest teilweiser) Rückhalt von Viren $\mathrm{zu}$ rechnen. Gelöste Ionen und Makromoleküle, wie beispielsweise $\mathrm{Hu}$ minstoffe oder Proteine können in der Regel mit Ultrafiltrationsanlagen nicht vollständig entfernt werden (Crittenden et al. 2012).

Im Gegensatz zu konventionellen Filteranlagen, die meist in Kesseln verbaut sind, zeichnen sich Membrananlagen durch eine weitaus komplexere Betriebsweise aus. Dazu gehören neben der eigentlichen Filtration des Rohwassers insbesondere auch eine etwa stündliche Rückspülung mit Filtrat zur Entfernung der abgesonderten Stoffe, und eine etwa tägliche Reinigung mit Lauge und Säure, um eine vollständige Regeneration der Membran zu gewährleisten. Die Intervalle und die Dauer dieser beiden Reinigungsschritte hängen jedoch stark von der jeweiligen Rohwasserqualität und Zusammensetzung ab und wirken sich auch auf die gesamte Ausbeute der Anlage aus (Crittenden et al. 2012).

Aufgrund der Komplexität der Betriebsweise, aber auch der unendlichen Vielfalt an Gewinnungsstellen für Trinkwasser ist es daher mehr als empfehlenswert, vor dem Bau einer Ultrafiltrationsanlage eine Pilotierung mit einer Versuchsanlage im kleineren Maßstab durchzuführen. Dadurch können einerseits die Intervalle und Dauer der Reinigungen und die Ausbeute abgeschätzt werden, aber andererseits auch verschiedene Betriebszustände der Anlage ausprobiert werden. Dabei kann beispielsweise das Verhalten der Anlage bei unterschiedlichen Zusammensetzungen des Rohwassers aufgrund der Nutzung verschiedener Ressourcen untersucht werden, oder Extremereignisse wie ein Hochwassereintrag oder der Ausfall einer Vorreinigungsstufe simuliert werden. Jedenfalls ist es ratsam, bei einer Pilotierung auch die denkbar ungünstigsten Zustände herbeizuführen, um die Grenzen der Anlage vorab auszuloten.

\subsection{Durchflusszytometrie und Biologische Stabilität}

Für die mikrobiologische Untersuchung von Trinkwasserproben haben sich in den vergangenen Jahren neben den klassischen Kultivierungsmethoden auch noch eine Reihe anderer Methoden etabliert. Eine dieser Methoden ist die sogenannte Durchflusszytometrie, deren großer Vorteil in der Fähigkeit liegt, Zellen unabhängig von ihrer Kultivierbarkeit zu detektieren. Es gibt Schätzungen, wonach sich ein Bruchteil (weniger als ein Prozent) aller in Wasserproben vorkommenden Bakterien auf klassischen Nährmedien kultivieren lassen.

Bei der Durchflusszytometrie werden Wasserproben mithilfe einer Hüllströmung zu einem so dünnen Flüssigkeitsfaden fokussiert, dass praktisch nur mehr einzelne bakterielle Zellen in diesem Flüssigkeitsfaden entlangfließen können. Durch Einsatz eines fluoreszierenden Farbstoffs, der spezifisch an die DNA der Mikroorganismen bindet, und eines Lasers können einzelne Zellen detektiert werden. Durch den Einsatz verschiedener Farbstoffe können wiederum unterschiedliche Eigenschaften untersucht werden - beispielsweise, ob es sich um lebende oder bereits abgestorbene Zellen handelt (ZunabovicPichler et al. 2018).

Ein Konzept, das in der Trinkwasserversorgung immer mehr zur Anwendung kommt, ist jenes der biologischen Stabilität. Um eine konstante Qualität des Wassers sicherzustellen, und verschiedene Prozesse in der Versorgungskette kontrollierbar zu machen, ist eine möglichst geringe Veränderung der mikrobiologischen Zusammensetzung wünschenswert. Insbesondere die Vermehrung und das Wachstum von Mikroorganismen, die sogenannte Wiederverkeimung, ist zu vermeiden. Damit eine Wiederverkeimung passieren kann, müssen zunächst lebensfähige Mikroorganismen vorhanden sein. Außerdem müssen ausreichende und geeignete Nährstoffe vorhanden sein, und das Wachstum darf durch Inhibitoren (z.B. Chlor) nicht verhindert werden (Prest et al. 2016).

Neben der sehr aufwendigen Bestimmung der Menge an assimilierbarem organischem Kohlenstoff (AOC) in einer Wasserprobe ist es auch möglich, das sogenannte Wachstumspotenzial zu bestimmen. Dabei werden Wasserproben unter definierten Bedingungen gelagert, und die Anzahl an Mikroorganismen zum Beispiel mithilfe der Durchflusszytometrie bestimmt. Je stärker die Zunahme an Zellen in diesen Lagerungsproben ist, umso geringer ist die biologische Stabilität der Wasserprobe einzuschätzen.

\section{Methodik}

\subsection{Experimentelle Herangehensweise}

Im Folgenden werden Ergebnisse aus einem Pilotierungsversuch einer Ultrafiltrationsanlage vorgestellt. Dabei wurden eine Vielzahl an Untersuchungen durchgeführt, die Betriebsweise und Ausbeute optimiert sowie die Eignung für weitere Aufbereitungsschritte definiert. In diesem Artikel soll aber insbesondere auf die Ergebnisse der durchflusszytometrischen Untersuchungen und der Wachstumspotenzialversuche eingegangen werden.

Der Pilotierungsversuch wurde über eine Dauer von 9 Monaten an einem Uferfiltratstandort in Österreich durchgeführt. Das Rohwasser wird aus einem Brunnenfeld mit 6 Brunnen gefördert, wobei die Brunnen aufgrund der Distanz zum Ufer unterschiedlich starken Einfluss des Oberflächengewässers erfahren. Aufgrund der Nähe zum Oberflächengewässer war insbesondere der Hochwasserfall und die damit einhergehende vermehrte Infiltration von Oberflächenwasser als Extrembelastung zu untersuchen.

Die Ultrafiltrationsanlage bestand aus einem Ultrafiltrationsmodul mit $80 \mathrm{~m}^{2}$ Membranoberfläche und $20 \mathrm{~nm}$ Porenweite, und wurde mit einem maximalen Durchfluss von $6,4 \mathrm{~m}^{3}$ pro Stunde im Dead-End-Modus betrieben. Die Anlage wurde vollautomatisiert betrieben, durch einen kleinen Vorhaltetank beschickt und belüftet. Die Rückspülung der Membran mit Filtrat erfolgte alle 45 bis 90 min und eine chemische Reinigung (CEB - chemical enhanced backwash) mit Lauge und Säure wurde alle 0,5 bis 5 Tage durchgeführt - jeweils in Abhängigkeit von der Rohwasserbeschaffenheit.

Die Pilotierung wurde in drei unterschiedlichen Phasen durchgeführt. Dabei wurden in der ersten Phase (03.07. bis 16.10.) alle 6 Brunnen des Brunnenfelds in beliebiger Weise betrieben und die Pilotierungsanlage langsam in ihrer Leistungsfähigkeit gesteigert. Nachdem sich ein stabiler Betrieb eingestellt hatte, wurden in Phase 2 (16.10 bis 22.11.) nur zwei, mit reduziertem Eisen belastete Brunnen genutzt. Abschließend wurde in Phase 3 (22.11. bis 31.03.) eine Hochwasserbelastung simuliert, indem dem Rohwasser 20 bis $40 \%$ des Oberflächenwassers zugemischt wurden. Um die Abtrennung der partikularen Stoffe in Phase 3 zu verbessern wurden je 
nach Belastung der Anlage zwischen 0,5 und 7 ppm Eisen-III-Chlorid als Flockungsmittel im Zulauf zur Membran zugegeben.

\subsection{Durchgeführte Untersuchungen}

Während der Pilotierung der Anlage wurde eine Vielzahl an chemisch-physikalischen und mikrobiologischen Untersuchungen durchgeführt, um die Aufbereitungsleistung der Anlage beurteilen zu können. Dazu zählten neben Temperatur, pH, Leitfähigkeit, Sauerstoffgehalt auch diverse Ionen, und die mikrobiologischen Standardparameter wie KBE, E. coli, Enterokokken usw. Für den weiteren Artikel werden aber nur drei Parameter näher betrachtet - der gelöste organische Kohlenstoff (DOC dissolved organic carbon), die durchflusszytometrischen Zellzahlen und das Wachstumspotenzial.

In zweiwöchigen Intervallen wurden Proben des Rohwassers, unmittelbar vor der Ultrafiltrationsmembran, und des Filtrats gezogen. Der organische Kohlenstoff wurde in einem externen Labor nach ISO-Standard ermittelt, während die durchflusszytometrischen Zellzahlen und das Wachstumspotenzial im mikrobiologischen Labor des Instituts für Siedlungswasserbau der Universität für Bodenkultur Wien bestimmt wurden.

Für die durchflusszytometrische Bestimmung der Zellzahlen wurde den Proben für die Totalzellzahlbestimmung mit dem Fluoreszenzfarbstoff SYBR-Green-I, bzw. für die Lebendzellzahlbestimmung ein Farbstoffgemisch aus Sybr-Green-I und Propidiumiodid zugegeben. Nach 15-minütiger Inkubation bei $37^{\circ} \mathrm{C}$ wurden im BD Accuri C6 Durchflusszytometer in jeweils $50 \mu \mathrm{l}$ die Anzahl an Events in dreifacher Wiederholung bestimmt. Durch Setzen entsprechender Gates (Zunabovic-Pichler et al. 2018) können diese Events als Zellen definiert und dadurch die Zellzahlen in den jeweiligen Proben ermittelt werden.

Für die Bestimmung des Wachstumspotenzials wurden vor Ort Proben in gläsernen Steilbrustflaschen abgefüllt. Zur Beseitigung jedweden organischen Kohlenstoffs aus den Flaschen wurden diese vorab für $5 \mathrm{~h}$ bei $550^{\circ} \mathrm{C}$ gemuffelt. Nach luftblasenfreier Abfüllung wurden die Flaschen gekühlt ins Labor transportiert und im Doppelansatz bei entweder $15^{\circ} \mathrm{C}$ oder $22^{\circ} \mathrm{C}$ in einem Inkubatorschrank gelagert.
Nach 7 und 14 Tagen wurden die Totalzellzahlen mittels Durchflusszytometrie bestimmt, wobei für diese beiden Untersuchungszeitpunkte jeweils eigene Flaschen untersucht wurden.

\section{Ergebnisse und Diskussion}

Im Zuge der Pilotierung konnten die gewünschten Erkenntnisse für den Betrieb der Anlage gewonnen werden, diese werden aber im weiteren Verlauf dieses Artikels nicht diskutiert. Im Besonderen ist jedoch anzumerken, dass die laut Trinkwasserverordnung zu untersuchenden, mikrobiologischen Parameter (Escherichia coli, Enterokokken, Pseudomonas aeruginosa, Clostridien und coliforme Bakterien) nicht mehr im Filtrat nachweisbar waren aber je nach Pegelstand des Oberflächengewässers durchaus im Rohwasser vorkamen (Daten nicht dargestellt).

In Abb. 1 ist die Verteilung der Konzentration des gelösten organischen Kohlenstoffs (DOC) nach Phase und Probenahmestelle dargestellt. Dabei ist zu erkennen, dass im Rohwasser der Pilotierungsphasen 1 und 2 mit einem für Grundwasser durchaus typischen DOC von unter $1 \mathrm{mg} / \mathrm{l} \mathrm{zu}$ rechnen war (Regan et al. 2017). Dieser DOC-Gehalt zeigt sich in weiterer Folge auch im Filtrat, was aufgrund der Porengröße der Membran auch zu erwarten ist, da Ultrafiltrationsanlagen in der Regel nicht in der Lage sind, gelöste Substanzen zu entfernen. In der Pilotierungsphase 3 hingegen, jene Phase, in der Oberflä-
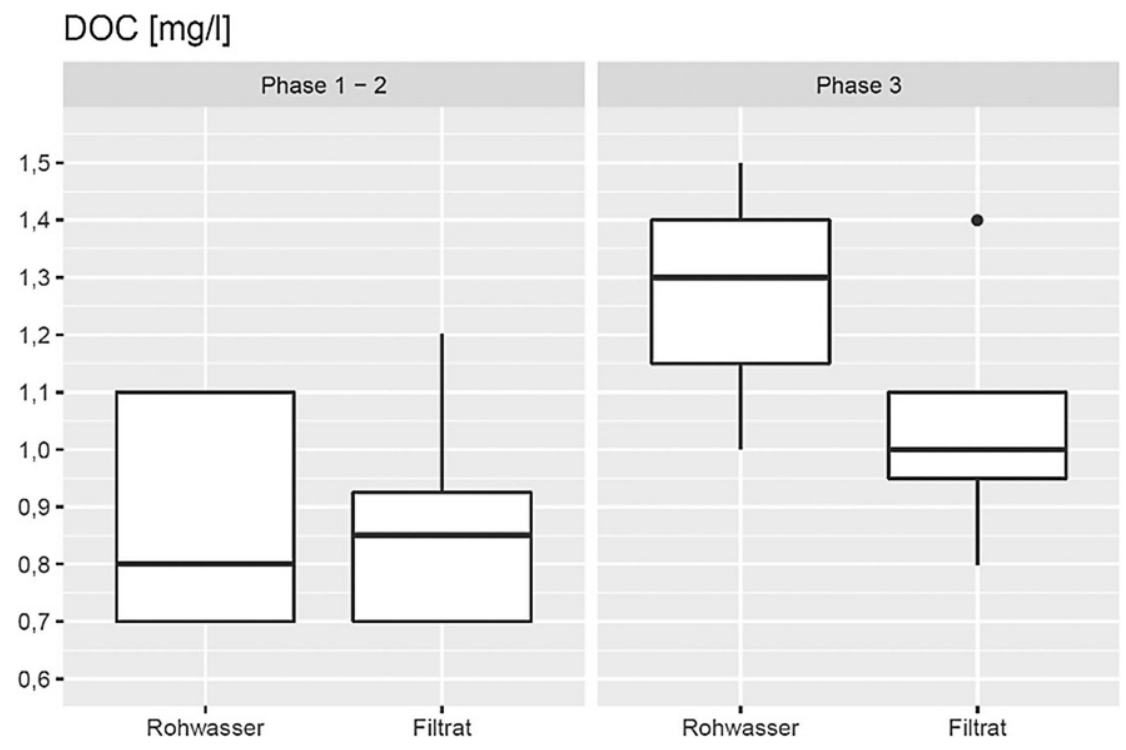

Abb. 1 Boxplot des gelösten organischen Kohlenstoffs (DOC), gruppiert nach Untersuchungsphase und Probenahmestelle

chenwasser hinzugeführt wurde, ist ein deutlicher Anstieg des DOC auf einen Wert von über $1 \mathrm{mg} / \mathrm{l} \mathrm{zu}$ erkennen. Betrachtet man das Mischungsverhältnis zwischen dem Uferfiltrat und dem Oberflächengewässer und berücksichtigt, dass Oberflächengewässer zwar einen weit höheren DOC-Gehalt aufweisen als Grundwässer, dieser aber starken saisonalen Schwankungen unterliegt und im Winter üblicherweise ein Minimum aufweist, lässt sich der verhältnismäßig geringe Anstieg zwischen den Phasen erklären (Mulholland und Hill 1997). Im Filtrat kommt es in Phase 3 ebenfalls zu einem Anstieg des DOC auf etwa $1 \mathrm{mg} / \mathrm{l}$, dieser fällt jedoch geringer aus als der Anstieg im Rohwasser. Diese sich dadurch ergebende Reduktion des DOC zwischen Rohwasser und Filtrat ist auf die Zugabe des Flockungsmittels zurückzuführen, da nicht nur partikuläre Stoffe, sondern auch gelöste Kohlenstoffverbindungen gebunden werden können (Matilainen et al. 2011)

Ein ähnliches Bild zeigt sich, wenn man die mit der Durchflusszytometrie ermittelten Zellzahlen betrachtet (Abb. 1 und 2). In den Phasen 1 und 2 sind die Zellzahlen im Rohwasser deutlich niedriger als in Phase 3, wo bis zu 1.500.000 Zellen pro Milliliter detektiert wurden. Dieser Umstand überrascht nicht weiter, da Oberflächenwässer typischerweise um etwa das 100-Fache höhere Zellzahlen aufweisen als Grundwässer (Griebler und Mösslacher 2003). Unmittelbar vor der Membran zeigt 
sich vor allem in Phase 3 eine leichte Reduktion der Zellzahlen. Diese kann möglicherweise auf die Verwendung von Flockungsmittel, und die damit einhergehende Aggregierung von Zellen zurückgeführt werden. Die Lebendzellzahlen sind dabei für alle untersuchten Stellen um etwa den Faktor 0,8 geringer als die Totalzellzahlen.

Trotz des hohen Ansteigens der Zellzahlen im Rohwasser zeigt sich in Phase 3 kein Anstieg der Zellzahlen im Filtrat. Dabei ist es wichtig anzumerken, dass die Zellzahlen im Filtrat nicht gegen 0 gehen, sondern knapp über der Bestimmungsgrenze der Durchflusszytometrie (500 Zellen pro Milliliter) liegen (Schweizerisches Lebensmittelbuch 2012).

Dieser Umstand ist umso bemerkenswerter, da die Membran eine Porenweite von $20 \mathrm{~nm}$ aufweist, also etwa $10 \times$ kleiner als die kleinsten bekannten Bakterien ist (Luef et al. 2015). Da die Membran den abschließenden Integrationstest ohne Probleme überstanden hat, ist es ausgesprochen unwahrscheinlich, dass die im Filtrat detektierten Zellen tatsächlich durch die Membran gelangt sind - außer es handelt sich um zum Beispiel sehr kleine Virenpartikel, deren Fluoreszenzsignal aber weitaus schwächer sein müsste (Huang et al. 2015). Da es jedoch sehr schwierig bis nahezu unmöglich ist, Wasserversorgungssysteme vollständig keimfrei zu halten, ist es unserer Meinung nach weitaus wahrscheinli- cher, dass die vorgefundenen Zellen aus filtratseitig vorhandenen Biofilmen stammen.

Die Ergebnisse der Wachstumspotenzialversuche sind in Abb. 3 dargestellt. Auch beim Wachstumspotenzial finden sich keine Unterschiede zwischen Phase 1 und 2, während sich in Phase 3 mit der Oberflächenwasserbeimischung ein völlig anderes Bild zeigt. Insgesamt ist die Wachstumsneigung an allen drei untersuchten Stellen in Phase 1 und 2 als gering einzustufen. Es zeigen sich vor allem im Rohwasser und vor der Membran nur geringe Erhöhungen der Zellzahlen nach 7 und 14 Tagen Lagerung. Diese geringen Wachstumsraten lassen, unter der Annahme dass kein Wachstumsinhibitor vorhanden ist, auf ein Wasser schließen, in dem zwar Mikroorganismen vorhanden sind (bis zu 300.000 Zellen pro Milliliter), aber die vorhandenen Nährstoffe bereits verbraucht wurden (Prest et al. 2016). Dadurch wäre kein weiterer Aufbau von Biomasse möglich, und die Zellzahlen bleiben unverändert.

Die Entwicklung in Phase 3 zeigt ein völlig anderes Bild. So kommt es im Rohwasser und vor der Membran nach den Probenahmen im Dezember zu einem moderaten Anstieg von etwa $50 \%$ gegenüber den Zellzahlen unmittelbar nach Probenahme. Ab Jänner hingegen, nehmen die Zellzahlen in diesen Proben im Verlauf des Wachstumsversuchs ab. Dieser Umstand kann möglicherweise dadurch erklärt werden, dass die in den Proben in hohen Zellzahlen vorkommenden Mikroorganismen aus dem Oberflächengewässer stammen, und dadurch an ebensolche Lebensbedingungen gewöhnt waren. Durch die Entnahme und die geänderten Bedingungen im Wachstumsversuch (kein Licht, keine Strömung, kein Sauerstoffeintrag) ist es wahrscheinlich, dass ein Teil der Mikroorganismen nicht weiter lebensfähig ist und abstirbt (Hunkeler und Goldscheider 2006).

Im Filtrat zeigt sich in Phase 3 hingegen durchgehend ein stark gestiegenes Wachstumspotenzial gegenüber Phase 1 und 2. Das Extrem zeigt sich am 18. Dezember mit einer etwa 1000fachen Zunahme an Zellen. Dieses Bild zeigt sich durchgehend bis zum Ende der durchgeführten Untersuchungen. Im Filtrat sind die Zellzahlen zwar nach 14 Tagen immer noch niedriger als ursprünglich im Rohwasser - trotzdem explodiert die Zellzahl im Filtrat relativ zur Ausgangszellzahl von etwa 500 Zellen pro Milliliter. Durch die Lagerung bei unterschiedlichen Temperaturen zeigt sich, dass das Wachstum im Filtrat bei niedrigeren Temperaturen $\left(15^{\circ} \mathrm{C}\right.$ gegenüber $22^{\circ} \mathrm{C}$ ) etwas verzögert stattfindet, was auf einen verlangsamten Stoffwechsel der Organismen bei niedrigeren Temperaturen zurückzuführen ist (Madigan et al. 2010).

Es ist anzumerken, dass dieses gesteigerte Wachstumspotenzial im Filtrat ohne eine nennenswerte Zunahme der Zellen zwischen Phase 1 und 2 bzw.

Vergleich Rohwasser, vor UF und Filtrat

Anzahl Messungen:

Phase 1 und $2: \mathrm{n}=6$

Phase 3: $n=11$

Farbstoff 追 s 追 SP

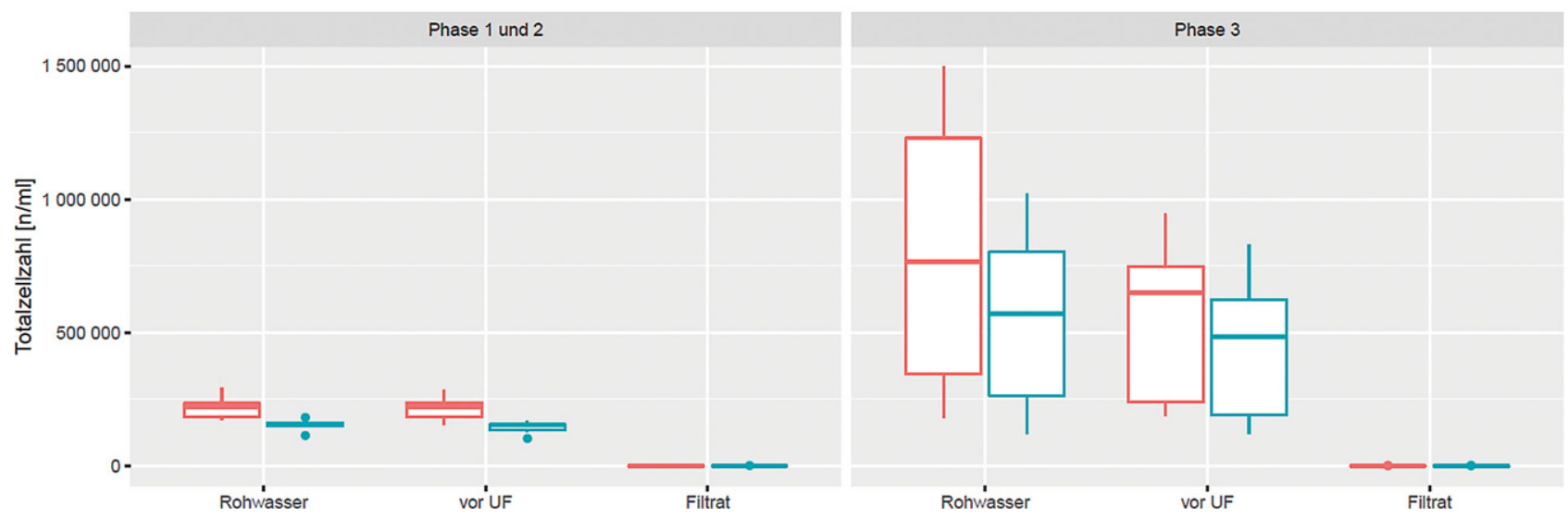

Abb. 2 Boxplot der durchflusszytometrischen Zellzahlen (Totalzellzahlen rot dargestellt, Lebendzellzahlen türkis), gruppiert nach Untersuchungsphase und Probenahmestelle 
Phase 3 (siehe Abb. 2) auftritt. Da das Vorhandensein von Wachstumsinhibitoren in Phase 1 und 2 ausgeschlossen werden kann, ist die Ursache wahrscheinlich vielmehr im Anstieg des verfügbaren Nährstoffdargebots zu suchen (Prest et al. 2016). Wie in Abb. 1 gezeigt wird, ist im Filtrat eine deutliche $\mathrm{Zu}$ nahme des organischen Kohlenstoffs in Phase $3 \mathrm{zu}$ erkennen. Ob Kohlenstoff tatsächlich der limitierende Nährstoff war, wurde zwar nicht untersucht, aber es ist anzunehmen, dass mit dem Oberflächenwasser auch andere Nährstoffe wie Stickstoff- oder Phosphorverbindungen in das System gelangen können, die durch die Ultrafiltration nicht zurückgehalten werden können.

\section{Schlussfolgerungen}

Durch die Pilotierung der hier untersuchten Ultrafiltrationsanlage konnten viele Erkenntnisse für eine großtechnische Anlage gewonnen werden. Diese
Ergebnisse wurden zwar nicht näher ausgeführt, betrafen aber insbesondere die Ermittlung einer voraussichtlichen Ausbeute der Anlage (Volumen an erzeugtem Filtrat abzüglich aufgewendetem Volumen für Rückspülung und Reinigung), Rückspülintervall und Rückspüldauer, die Effizienz und Notwendigkeit chemischer Reinigungen, und den Einsatz von Flockungsmittel. Insbesondere in Phase 3, der simulierten Hochwassersituation durch Oberflächenwasserbeimischung, konnten wichtige Erkenntnisse über die Leistungsfähigkeit der Anlage im Extremfall gewonnen werden.

Bezüglich der mikrobiologischen Reinigungsleistung hat die Pilotierung auch unter Belastung die wesentlichen Indikatororganismen (nicht im Sinne der Parameter mit Indikatorfunktion, sondern im Sinne Fäkalindikatoren) wie E. coli, Enterokokken oder coliforme Bakterien, vollständig zurückgehalten. Interessanterweise sind die mit- tels Durchflusszytometrie ermittelten Zellzahlen im Filtrat trotz einer etwa zehnfach kleineren Porenweite der Membran nicht gegen Null gegangen. Wahrscheinlich ist diese Beobachtung auf filtratseitige Verunreinigungen im System zurückzuführen. Dieser Umstand ist sinnbildlich für Trinkwassersysteme, die nie als vollständig sterile Systeme verstanden werden können.

Es konnte gezeigt werden, dass das Vorhandensein geringer Mengen an Mikroorganismen nach der Membran, in Zusammenhang mit gesteigerter Nährstoffverfügbarkeit im Filtrat, zu einer Reduktion der Biostabilität führen kann. Insbesondere im Extremfall mit hohem Oberflächenwassereintrag werden zwar die Mikroorganismen des Oberflächenwassers zurückgehalten, die niedermolekularen gelösten organischen Verbindungen können aber die Membran passieren. Diese leicht verfügbaren Substanzen stehen den Mikroorganismen, deren Auftreten im

Rohwasser - vor UF - Filtrat: Zellen pro ml

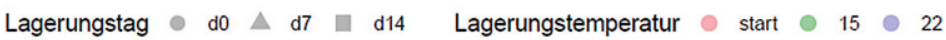
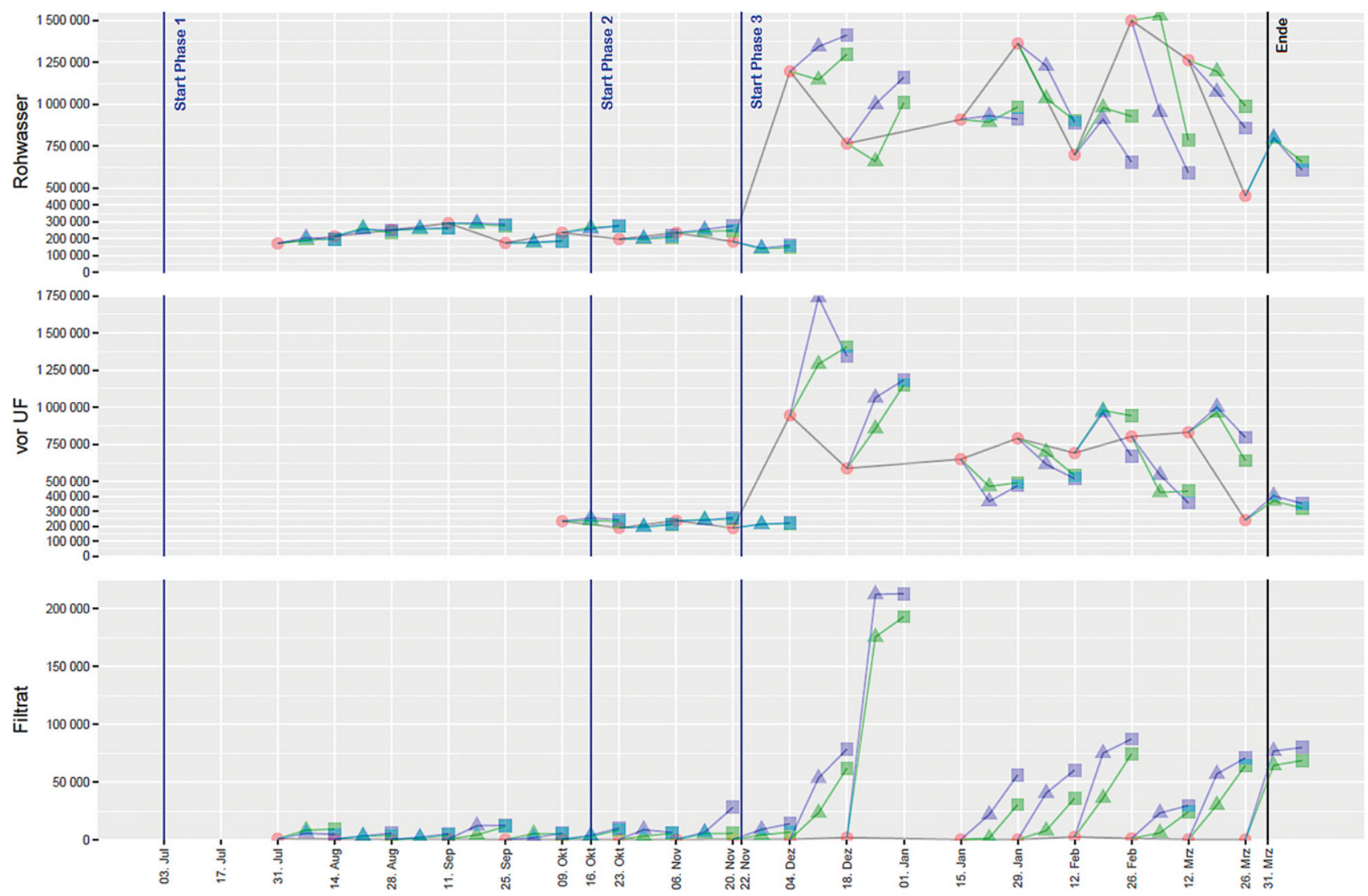

Abb. 3 Darstellung derWachstumsversuche-diegrünen (Lagerung bei $15^{\circ} \mathrm{C}$ ) bzw. dieblauen Punkte $\left(22^{\circ} \mathrm{C}\right)$ geben dieEntwicklung nach Lagerung wieder 
Filtratbereich praktisch nicht zu verhindern ist, als Grundlage für eine bis zu 1000-fache Vermehrung zur Verfügung.

Um ein biologisch stabiles System zu schaffen, könnte es im vorliegenden Fall daher notwendig werden, das Nährstoffdargebot - zum Beispiel durch eine Oxidierung und/oder Aktivkohlefiltration - zumindest im Hochwasserfall weiter $\mathrm{zu}$ reduzieren. Jedenfalls können durch eine Pilotierung und die entsprechenden Untersuchungen $\mathrm{Zu}$ sammenhänge aufgezeigt werden, die im weiteren Versorgungssystem zu Herausforderungen werden können. Aufgrund der im Vorhinein gewonnenen Erkenntnisse können dadurch bereits vorab Maßnahmen geplant werden, die ein Aufwachsen von Mikroorganismen verhindern und ein biologisch stabiles System gewährleisten.
Funding Open access funding provided by University of Natural Resources and Life Sciences Vienna (BOKU).

\section{Interessenkonflikt}

P. Proksch, D. J. Kerschbaumer, C. Schönher, M. Zunabovic-Pichler, E. Mayr und R. Perfler geben an, dass kein Interessenkonflikt besteht.

Open Access Dieser Artikel wird unter der Creative Commons Namensnennung 4.0 International Lizenz veröffentlicht, welche die Nutzung, Vervielfältigung, Bearbeitung, Verbreitung und Wiedergabe in jeglichem Medium und Format erlaubt, sofern Sie den/die ursprünglichen Autor(en) und die Quelle ordnungsgemäß nennen, einen Link zur Creative Commons Lizenz beifügen und angeben, ob Änderungen vorgenommen wurden.

Die in diesem Artikel enthaltenen Bilder und sonstiges Drittmaterial unterliegen ebenfalls der genannten Creative Commons Lizenz, sofern sich aus der Abbildungslegende nichts anderes ergibt. Sofern das betreffende Material nicht unter der genannten Creative Commons Lizenz steht und die betreffende Handlung nicht nach gesetzlichen Vorschriften erlaubt ist, ist für die oben aufgeführten Weiterverwendungen des Materials die Einwilligung des jeweiligen Rechteinhabers einzuholen.

Weitere Details zur Lizenz entnehmen Sie bitte der Lizenzinformation auf http://creativecommons.org/licenses/ by/4.0/deed.de.

\section{Literatur}

Crittenden, J. C., Trussell, R. R., Hand, D. W., Howe, K. J., \& Tchobanoglous, G. (2012): MHW's Water Treatment. John Wiley \& Sons., Hoboken, New Jersey

DVGW W 213-5 (2019): Filtrationsverfahren zur Partikelentfernung - Teil 5: Membranfiltration. Technische Regel - Arbeitsblatt., Deutscher Verein des Gas- und Wasserfaches e. V., Bonn Griebler, C., \& Mösslacher, F. (2003): Grundwasser-Ökologie. Facultas Verlag, Wien.

Huang, X., Min, J. H., Lu, W., Jaktar, K., Yu, C., \& Jiang, S. C. (2015): Evaluation of methods for reverse osmosis membrane integrity monitoring for wastewater reuse. Journal of Water Process Engineering, 7, 161-168.

Hunkeler, D., \& Goldscheider, N. (2006): BiozÖnosen im Grundwasser - Grundlagen und Methoden der Charakterisierung von mikrobiellen Gemeinschaften. Umwelt-Wissen Nr. 0603, Bundesamt Für Umwelt, Bern.

Luef, B., Frischkorn, K. R., Wrighton, K. C., Holman, H.-Y. N., Birarda, G., Thomas, B. C., Singh, A., Williams, K. H., Siegerist, C. E., Tringe, S. G. Downing, K. H., Comolli, L. R., \& Banfield, J. F (2015): Diverse uncultivated ultra-small bacte- rial cells in groundwater. Nature Communications, 6, 6372 .

Madigan, M. T., Martinko, J. M., Stahl, D., \& Clark, D. P. (2010): Brock Biology of Microorganisms (13th Edition) (13th ed.). Benjamin Cummings.

Matilainen, A., Gjessing, E. T., Lahtinen, T. Hed, L., Bhatnagar, A., \& Sillanpää, M. (2011) An overview of the methods used in the characterisation of natural organic matter (NOM) in relation to drinking water treatment. Chemosphere, 83(11), 1431-1442.

Mulholland, P. J., \& Hill, W. R. (1997): Seasona patterns in streamwater nutrient and dissolved organic carbon concentrations: Separating catchment flow path and in-stream effects. Water Resources Research, 33(6), 1297-1306.

Prest, E. I., Hammes, F., van Loosdrecht, M C. M., \& Vrouwenvelder, J. S. (2016): Biological stability of drinking water: Controlling factors, methods, and challenges. Frontiers in Microbiology, 7(FEB), 1-24

Regan, S., Hynds, P., \& Flynn, R. (2017): An overview of dissolved organic carbon in groundwater and implications for drinking water safety. Hydrogeology Journal, 25(4), 959-967.
Schweizerisches Lebensmittelbuch (2012): Bestimmung der Totalzellzahl und des quantitativen Verhältnisses der Zellen niedrigen bzw. hohen Nukleinsäuregehaltes in Süsswasser mittels Durchflusszytometrie. Schweizerisches Lebensmittelbuch, 1-30.

Zunabovic-Pichler, M., Mayr, E., Perfler, R. Schönher, C., Fiedler, C., Kerschbaumer, D., \& Proksch, P. (2018): Durchflusszytometrie in der Wasserversorgung-Mikrobiologische Charakterisierung von Einflussfaktoren und Zustandsveränderungen bei der Wasserversorgung. Bundesministerium für Nachhaltigkeit und Tourismus, Wien

Hinweis des Verlags Der Verlag bleibt in Hinblick auf geografische Zuordnungen und Gebietsbezeichnungen in veröffentlichten Karten und Institutsadressen neutral. 